\title{
Häufigkeit psychischer Komorbiditäten in der stationären pneumologischen Rehabilitation und Indikationsstellung zu psychol. Beratung/Psychotherapie
}

Incidence of Psychic Comorbidity in Inpatient Pneumological Rehabilitation

and Indication for Psychological Counselling/Psychotherapy

Autoren

Institut
M. Schwiersch ${ }^{1}$, K. Hartl ${ }^{1}$, K. Schultz ${ }^{1}$, H. Reinecker ${ }^{2}$

Fachklinik Allgäu (Chefarzt Dr. med. K. Schultz)

Universität Bamberg (Prof. Dr. H. Reinecker) eingereicht 15. 9. 2005

akzeptiert 10.4. 2006.

\section{Bibliografie}

Dol $10.1055 / \mathrm{s}-2006-932208$

Pneumologie 2005; 60; 474-479

(c) Georg Thieme Verlag KC

Stuttgart $\cdot$ New York

ISSN 0934-8387

Korrespondenzadresse

Dipl. Psych., Dr. biol. hum. Martin Schwiersch

Fachklinik Allgäu

Peter-Heel-Str. 29

87459 Pfronten-Ried

mschwiersch@ahg.de

\section{Zusammenfassung}

Die vorliegende Arbeit untersucht psychische Komorbiditäten von Patienten mit chronischen Erkrankungen der Atmungsorgane in stationärer pneumologischer Rehabilitation und die Indikationsstellung zu psychologischer Beratung/Psychotherapie. Sie will damit einen Beitrag leisten, psychologische und psychotherapeutische Hilfen in stationärer pneumologischer Rehabilitation rational zu begründen. Gemäß anerkannter psychiatrischer Screening-Skalen (BSI, HADS) erweisen sich 30\% der 159 untersuchten Reha-Patienten in beiden Fragebogen (BSI und HADS-D) als auffällig, weitere $51 \%$ sind in einem der Fragebogen als auffällig einzuschätzen. Ca. 30\% der pneumologischen Patienten der Fachklinik Allgäu werden ärztlicherseits zu psychologischer Beratung/Psychotherapie indiziert. Die Indikationsstellung erweist sich vor diesem Hintergrund als valide. Die Gruppe der trotz vorliegender psychischer Beeinträchtigung nicht einer psychologischen Beratung/Psychotherapie zugeführten Patienten zeigt signifikant geringere Behandlungsmotivation und geringeren psychischen Leidensdruck. Das hohe Ausmaß psychischer Belastungen belegt die Relevanz psychologischer bzw. psychotherapeutischer Hilfen in pneumologischer Rehabilitation.

\section{Einleitung}

Die rechtzeitige Erkennung und angemessene Behandlung psychischer Störungen hat große Bedeutung für den Verlauf von körperlichen Erkrankungen. Bengel \& Jäckel (2002) [1] fassen die Bedeutung komorbider psychischer Probleme bei körperlich Kranken zusammen: „Komorbide psychische Probleme erhöhen die somatische

\section{Abstract}

The present study examines psychic comorbidities in patients with chronic diseases of the airways in pneumological rehabilitation centres and the indication for psychological counselling/ psychotherapy. It is thus intended to help provide a rational basis for psychological and psychotherapeutic help in inpatient pneumological rehabilitation. According to accepted psychiatric screening scales (BSI, HADS), $30 \%$ of the 159 examined patients undergoing rehabilitation were conspicuous in both questionnaires (BSI and HADS-D) and further $51 \%$ were considered as conspicuous in one of the questionnaires. About $3 \%$ of the pneumological patients in the Allgäu centre had a medical indication for psychological counselling/psychotherapy. In this respect the indication proved to be valid. The group of patients who were not referred to a psychological counselling/ psychotherapy in spite of an existing psychic impairment showed a significantly lower motivation for treatment and a lower psychic tolerance. The large proportion of psychic stress confirms the relevance of psychological and/or psychotherapeutic help in pneumological rehabilitation.
Morbidität und Mortalität, führen zu höherer Inanspruchnahme und damit zu erhöhten Kosten im medizinischen Versorgungssystem“.

Dies vorausgesetzt, ergibt sich auch für die pneumologische Rehabilitation die Aufgabe, begleitende psychische Störungen zu identifizieren und einer angemessenen Behandlung zuzuführen $[2,3,4]$. Derzeit kann die Frage des Ausmaßes und der Art begleitender psychischer Störungen 
bei pneumologischen Patienten sowie der Notwendigkeit der Indikationsstellung zu psychologischen und psychotherapeutischen Hilfen empirisch nicht zufriedenstellend beantwortet werden, wenngleich sich zunehmend der Blick auf diese Frage richtet $[5,6]$. Verschiedene Autoren berichten über relevante Prävalenzraten psychischer Störungen bei pneumologischen Patienten, z. B. [5, 6, 7,8, 9]. Härter u. Mitarb. [6,13] kommen in ihrer Studie über Prävalenz und Art psychischer Störungen in der medizinischen Rehabilitation zum Schluss, dass jeder dritte Rehabilitand in der somatischen Rehabilitation (untersuchte Indikationsbereiche: Orthopädie, Kardiologie, Onkologie) eine erhöhte psychische Belastung aufweist, wobei affektive und Angststörungen im Vordergrund stehen.

Die vorliegende Studie widmet sich folgenden Fragestellungen:

- In welchem Ausmaß liegen bei Patienten mit chronischen Erkrankungen der Atmungsorgane in stationärer Rehabilitation psychische Belastungen oder Komorbiditäten vor?

- Wie wirken sich Behandlungsmotivation und psychischer Leidensdruck der Patienten auf die Indikationsstellung zu psychologischen Hilfen aus?

- Gibt es einen Zusammenhang zwischen testdiagnostisch erhobener psychischer Belastung und klinischer Belastungseinschätzung seitens des behandelnden Arztes?

Durch Berücksichtigung dieser Faktoren soll langfristig eine rationale Begründung psychologischer Hilfen/Psychotherapie in pneumologischer Rehabilitation geleistet werden, da nach Bengel \& Jäckel (2002) [1] derzeit „in der Rehabilitationspraxis (...) ein großer Teil der psychisch beeinträchtigten Patienten und Patientinnen weder diagnostiziert noch ausreichend behandelt [wird]“.

Ein Screening psychischer Belastungen und Komorbiditäten stellt eine notwendige Basis für entsprechende Indikationsentscheidungen in stationärer pneumologischer Rehabilitation dar. Vorrangiges Ziel der vorliegenden Arbeit ist die Deskription des Ausmaßes psychischer Belastung und des psychologischen Beratungs-/psychotherapeutischen Behandlungsbedarfs und damit eine Validitätsprüfung der Indikationsstellung zu psychologischen Hilfen (Beratung, Psychotherapie), die in der Fachklinik Allgäu, an der die vorliegende Studie durchgeführt wurde, durch das klinische Urteil des behandelnden Arztes erfolgt.

Für die vorliegende Studie stellt sich neben der Deskription von Bedarf und Indikationsstellung zusätzlich die Frage, welche Faktoren den Prozess der Indikationsstellung beeinflussen.

\section{Material und Methoden}

Die Studie strebte an, etwa 15\% der pneumologischen Patienten der Fachklinik Allgäu eines Jahres zu rekrutieren. Um den sich ergebenden Stichprobenumfang von ca. 200 Patienten zu erreichen, wurden alle stationären pneumologischen Rehabilitationspatienten der Fachklinik Allgäu im Zeitraum 14. 4. 03-4. 7. 03 in die Stichprobe aufgenommen, soweit sie in der Lage waren, die Fragebogenbatterie vollständig auszufüllen.

Zur Abschätzung des psychologischen Beratungs- und/oder psychotherapeutischen Behandlungsbedarfs sind vor allem solche Messinstrumente geeignet, die Schwellenwerte für die Einschätzung der psychischen Belastung der Patienten angeben. Im Rahmen eines Screenings ist damit eine relativ schnelle Unterscheidung zwischen wahrscheinlich gesunden und möglicherweise erkrankten Personen möglich [10].
Als Screeningverfahren wurden das Brief Symptom Inventory (BSI) [11] und die HADS-D [12] ausgewählt. Das BSI erfasst die subjektiv empfundene Beeinträchtigung durch körperliche und psychische Symptome. Sie stellt eine Kurzform der SymptomCheckliste SCL-90-R dar und erfasst die psychische Belastung in 9 Skalen (u.a. Depressivität, Ängstlichkeit, Zwanghaftigkeit, Somatisierung) sowie in drei globalen Kennwerten. Die HADS-D wurde zum gezielten Einsatz zur Erfassung von Angst und Depressivität bei somatischen Patienten entwickelt. Angst und Depressivität werden mit je 7 Items erfasst. Indikationsstellung, Behandlungsmotivation und psychischer Leidensdruck wurden in einem von den Autoren entwickelten Rating von den behandelnden Ärzten und Psychologen erfragt. In die Studie wurden weitere Informationen zur pneumologischen Diagnose sowie eine Einschätzung des Schweregrades der Erkrankung aufgenommen.

Ausgehend von Korrelationsanalysen wurden auffällige Werte in den beiden Screeninginstrumenten BSI und HADS [11,12] nach dem in Tab. 1 dargestellten Algorithmus zu dem Kennwert „Psychischer Status" zusammengefasst, der in 3 Abstufungen zwischen „unbelastet“ (0) bis „deutlich komorbid“ (2) unterscheidet.

Tab. 1 zeigt die Operationalisierung der Auffälligkeit in den beiden Fragebögen, während Tab. 2 die Operationalisierung des Kennwertes Psychischer Status - der sich aus diesen beiden Fragebogen zusammensetzt - beschreibt.

\section{Tab. 1 Schwellenwerte der verwendeten Screeninginstrumente

\begin{tabular}{|lll|}
\hline & Unauffällig (0) & Auffällig (1) \\
\hline HADS-D Angst & $0-10$ & $11-21$ \\
\hline HADS-D Depression & $0-8$ & $9-21$ \\
\hline HADS-D gesamt & $\begin{array}{l}\text { HADS-D Depression } \\
\text { oder HADS-D Angst } \\
\text { auffällig }\end{array}$ \\
\hline BSI & $\mathrm{T}_{\text {GSI }} \geq 63$ oder \\
(Franke, 2000, S. 20) & $\mathrm{T}_{25 k a l e n} \geq 63$ \\
\hline $\begin{array}{l}\text { Die Normwerte zur Berechnung der T-Werte im BSI sind der Normtabelle für } \\
\text { Erwachsene im Manual von Franke (2000) [11] entnommen (S. 93 ff). }\end{array}$ \\
$\begin{array}{l}\text { Da BSI nur eine Aufteilung ja (1)/nein (0) und nicht hinsichtlich vielleicht } \\
\text { (0,1,2) vorsieht, wurde in Anlehnung an eine von Frau Reusch in Absprache }\end{array}$ \\
$\begin{array}{l}\text { mit Herrn PD Dr. Hermann erstellte Syntax für die HADS-D ebenfalls nur eine } \\
\text { dichotome Erfassung vorgenommen [12]. }\end{array}$
\end{tabular}

\section{Statistische Methode}

Neben deskriptiven Statistiken werden Zusammenhangsmaße und Unterschiedsprüfungen gerechnet. Da für die Kennwerte Psychischer Status, Behandlungsmotivation und Leidensdruck nicht von einem Intervallniveau der Daten ausgegangen werden kann, werden bei diesen Parametern der $\mathrm{X}^{2}$-Test bzw. der MannWithney-U-Test herangezogen.

\section{Ergebnisse}

\section{Stichprobenbeschreibung}

In die Stichprobe gingen 193 Probanden ein, davon wurden 47 von Psychologen gesehen, dies entspricht einem Prozentsatz von 24\%. 159 füllten die Fragebogenbatterie aus. 34 Non-Responder $(18 \%)$ sind zu verzeichnen. 
Tab. 2 Algorithmus zur Operationalisierung des Kennwerts „Psychischer Status“

\begin{tabular}{|c|c|c|c|}
\hline BSI unauffällig & $\begin{array}{l}\text { HADS-D Angst } \\
\text { unauffällig }\end{array}$ & $\begin{array}{l}\text { HADS-D De- } \\
\text { pression } \\
\text { unauffällig }\end{array}$ & $\begin{array}{l}\text { Psychischer } \\
\text { Status } \\
\text { (0) }\end{array}$ \\
\hline BSI unauffällig & $\begin{array}{l}\text { HADS-D Angst } \\
\text { unauffällig }\end{array}$ & $\begin{array}{l}\text { HADS-D De- } \\
\text { pression } \\
\text { auffällig }\end{array}$ & $\begin{array}{l}\text { Psychischer } \\
\text { Status } \\
\text { (1) }\end{array}$ \\
\hline BSI unauffällig & $\begin{array}{l}\text { HADS-D Angst } \\
\text { auffällig }\end{array}$ & $\begin{array}{l}\text { HADS-D De- } \\
\text { pression } \\
\text { unauffällig }\end{array}$ & $\begin{array}{l}\text { Psychischer } \\
\text { Status } \\
\text { (1) }\end{array}$ \\
\hline BSI unauffällig & $\begin{array}{l}\text { HADS-D Angst } \\
\text { auffällig }\end{array}$ & $\begin{array}{l}\text { HADS-D De- } \\
\text { pression } \\
\text { auffällig }\end{array}$ & $\begin{array}{l}\text { Psychischer } \\
\text { Status } \\
\text { (1) }\end{array}$ \\
\hline BSI auffällig & $\begin{array}{l}\text { HADS-D Angst } \\
\text { unauffällig }\end{array}$ & $\begin{array}{l}\text { HADS-D De- } \\
\text { pression } \\
\text { unauffällig }\end{array}$ & $\begin{array}{l}\text { Psychischer } \\
\text { Status } \\
\text { (1) }\end{array}$ \\
\hline BSI auffällig & $\begin{array}{l}\text { HADS-D Angst } \\
\text { unauffällig }\end{array}$ & $\begin{array}{l}\text { HADS-D De- } \\
\text { pression } \\
\text { auffällig }\end{array}$ & $\begin{array}{l}\text { Psychischer } \\
\text { Status } \\
\text { (2) }\end{array}$ \\
\hline BSI auffällig & $\begin{array}{l}\text { HADS-D Angst } \\
\text { auffällig }\end{array}$ & $\begin{array}{l}\text { HADS-D De- } \\
\text { pression } \\
\text { unauffällig }\end{array}$ & $\begin{array}{l}\text { Psychischer } \\
\text { Status } \\
\text { (2) }\end{array}$ \\
\hline BSI auffällig & $\begin{array}{l}\text { HADS-D Angst } \\
\text { auffällig }\end{array}$ & $\begin{array}{l}\text { HADS-D De- } \\
\text { pression } \\
\text { auffällig }\end{array}$ & $\begin{array}{l}\text { Psychischer } \\
\text { Status } \\
\text { (2) }\end{array}$ \\
\hline
\end{tabular}

Der Kennwert „Psychischer Status“ gibt das Maß auffälliger Werte in den verwendeten Screeningskalen an.

$0=$ nicht auffällig: unauffällige Werte in allen drei Skalen

$1=$ tendenziell auffällig:

- unauffällige Werte im BSI und auffällige Werte in einer der beiden oder beiden Skalen der HADS

- auffällige Werte im BSI und unauffällige Werte in beiden Skalen der HADS

2 = auffällig: auffällige Werte im BSI und in einer der beiden oder beiden

Skalen der HADS

Die Altersspanne der Probanden liegt zwischen 32 und 90 Jahren; der Mittelwert beträgt 66 Jahre (SD 10,0). Mit einem Anteil von $62 \%$ sind mehr weibliche Patienten vertreten.

Abb. 1 zeigt die Verteilung der pneumologischen Diagnosen in der untersuchten Stichprobe.

COPD und Asthma bronchiale stellen mit 44\% resp. 35\% die häufigsten Diagnosen dar. Unter ,sonstige pneumologische Diagnose“ (17,4\%) fallen u.a. chronische Sinusitiden, Lungenfibrose, Z. n. Pneumonien, Atempumpenschwäche.

Die Patienten, die die Fragebogenbatterie ausgefüllt haben, unterscheiden sich nicht hinsichtlich der Indikationsstellung von den Non-Respondern.

Behandlungsbedarf, psychischer Leidensdruck, Behandlungsmotivation und Indikationsstellung

31 \% der Patienten werden von den Ärzten als psychologisch behandlungsbedürftig eingeschätzt, und bei $29 \%$ der Patienten erfolgt eine Indikationsstellung zu psychologischer Beratung/Psychotherapie. Bei annähernd allen als behandlungsbedürftig eingeschätzten Patienten erfolgt eine Indikationsstellung zu psychologischer Beratung/Psychotherapie $\left(\mathrm{X}^{2}\right.$-Test, $\left.\mathrm{p}=0,000\right)$.

Von den 47 von Psychologen betreuten Patienten werden 79\% von diesen als eindeutig beratungsbedürftig/psychotherapiebedürftig angesehen. Bei 19\% liegt nach ihrer Einschätzung ein optionaler Beratungsbedarf vor und $2 \%$ der Patienten zeigen nach psychologischer Einschätzung keinen Beratungsbedarf.

Ärzte und nachfolgend behandelnde Psychologen sehen bei den indizierten Patienten übereinstimmend einen deutlichen psychischen Leidensdruck.

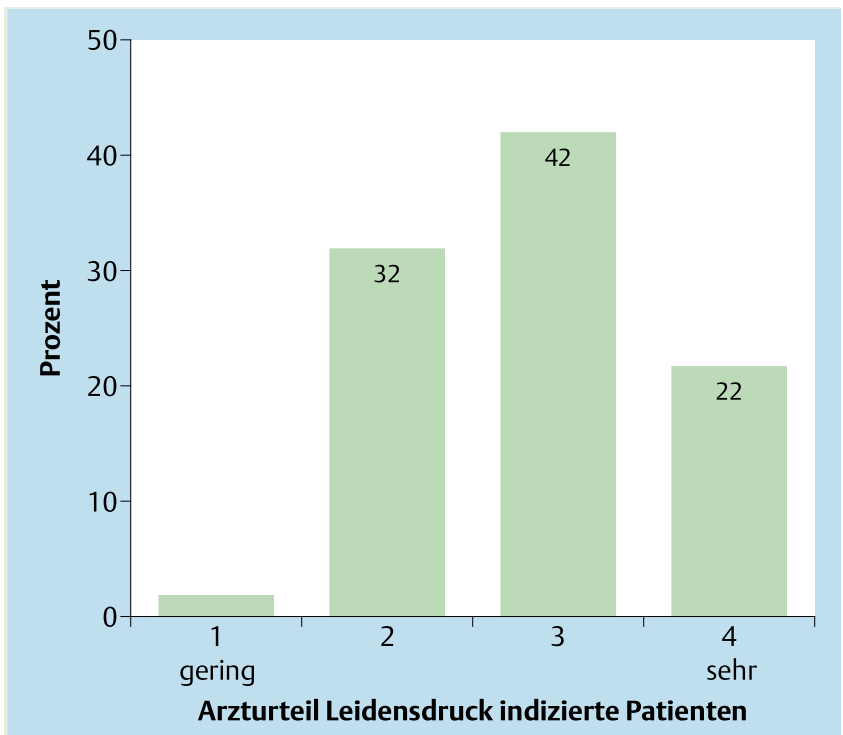

Abb. 1 Pneumologische Diagnosen in der Stichprobe.

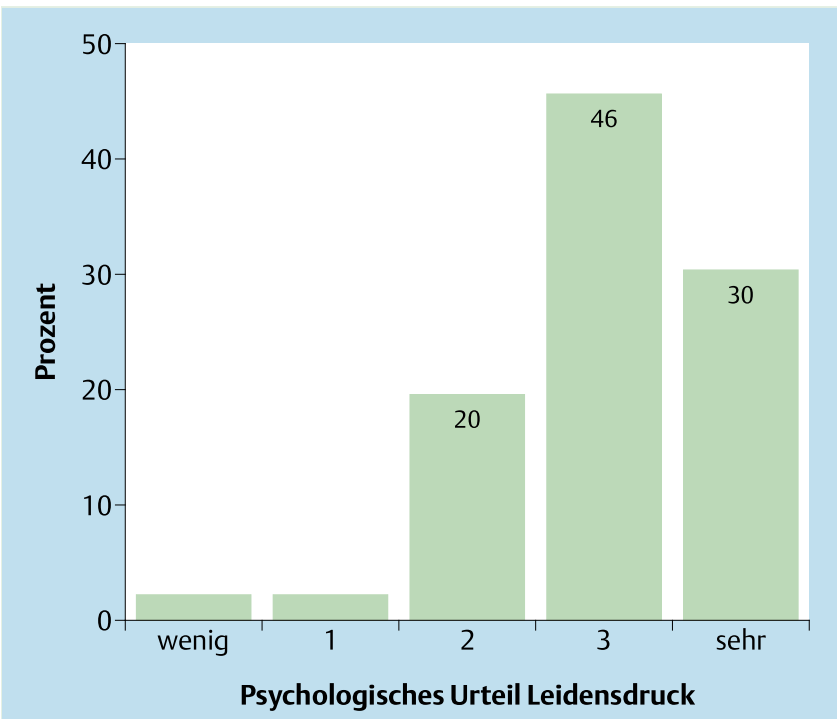

Abb. 2 Verteilung der ärztlichen Einschätzung des psychischen Leidensdrucks für die indizierten Patienten.

Die Abb. $\bullet 2$ und $\bullet \mathbf{3}$ zeigen die ärztliche bzw. psychologische Einschätzung der Verteilung des psychischen Leidensdrucks, Abb. 4 und 5 die ärztliche bzw. psychologische Einschätzung der Verteilung der Behandlungsmotivation.

Für die Gruppe der Patienten, die neben der ärztlichen Betreuung auch psychologisch gesehen wurde, besteht zwischen den Einschätzungen der beiden Fachgruppen von Leidensdruck und Behandlungsmotivation ein hoher und signifikanter Zusammenhang (Kontingenzkoeffizient $=0,526 ; \mathrm{p}=0,026$ resp. 0,643; $\mathrm{p}=0,002)$. 


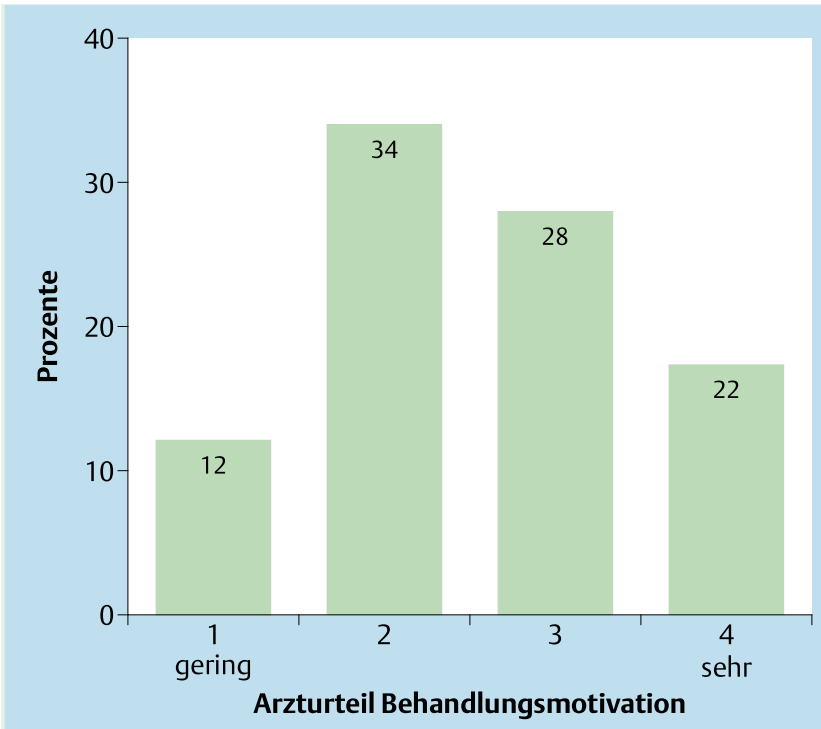

Abb. 3 Verteilung der psychologischen Einschätzung des Leidensdrucks.

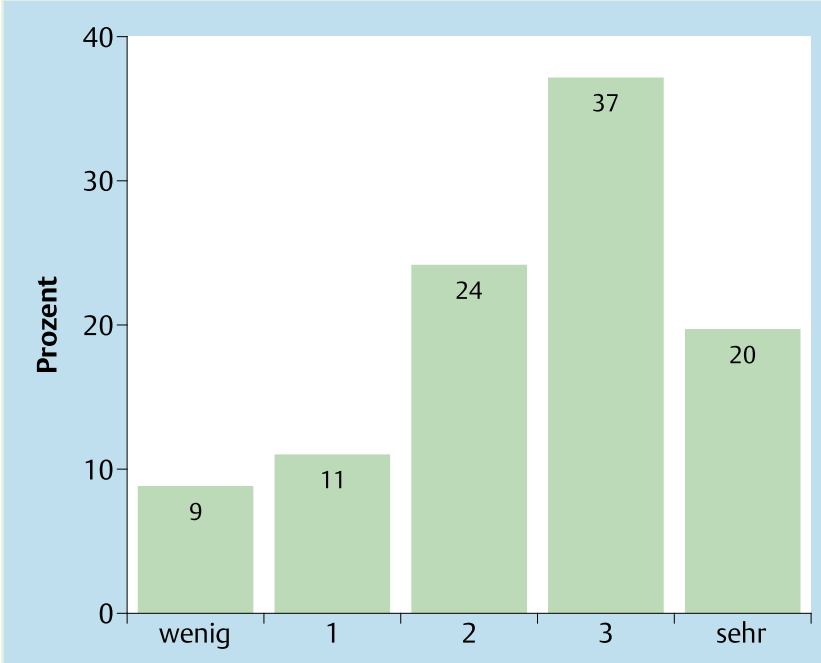

Psychologisches Urteil Behandlungsmotivation

Abb. 4 Verteilung der ärztlichen Einschätzung der Behandlungsmotivation bei indizierten Patienten.

\section{Zusammenhänge zwischen Bedarf, psychischem Leidensdruck, Behandlungsmotivation und Indikationsstellung}

Die behandelnden Ärzte sehen bei den indizierten Patienten einen deutlich höheren Leidensdruck und eine deutlicher vorliegende Behandlungsmotivation als bei der Gesamtstichprobe der pneumologischen Patienten.

Tab. 3 zeigt die Mittelwertsunterschiede in Leidensdruck und Behandlungsmotivation zwischen indizierten und nicht-indizierten Patienten 1 .

\footnotetext{
${ }^{1}$ Gemeint sind Patienten, bei denen ärztlich eine Indikationsstellung zu psychologischer Beratung/Psychotherapie erfolgte (,indiziert“) bzw. nicht erfolgte („nicht indiziert“).
}

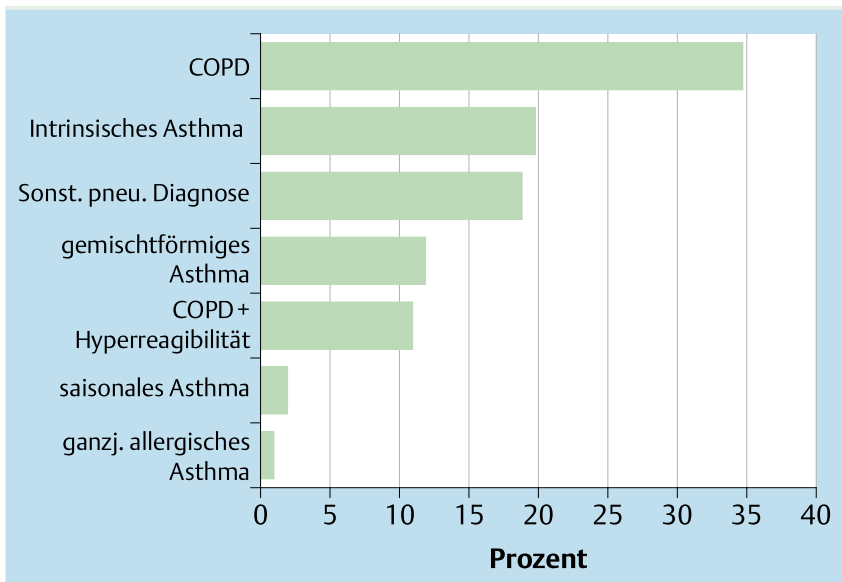

Abb.5 Verteilung der psychologischen Einschätzung der Behandlungsmotivation.

Tab. 3 Mittelwertsunterschiede in psychischem Leidensdruck und Behandlungsmotivation zwischen indizierten und nicht indizierten Patienten

\begin{tabular}{|l|lll|}
\hline & $\begin{array}{l}\text { Indizierte } \\
\text { Patienten }\end{array}$ & $\begin{array}{l}\text { Nicht-indizierte } \\
\text { Patienten }\end{array}$ & $\begin{array}{l}\text { Signifi- } \\
\text { kanz }\end{array}$ \\
\hline Leidensdruck & 2,68 & 0,97 & 0,000 \\
\hline $\begin{array}{l}\text { Behandlungsmo- } \\
\text { tivation }\end{array}$ & 2,48 & 0,64 & 0,000 \\
\hline
\end{tabular}

Tab. 4 Korrelative Zusammenhänge zwischen psychischem Leidensdruck, Behandlungsbedarf, Behandlungsmotivation und Indikationsstellung

\begin{tabular}{|c|c|c|c|}
\hline & $\begin{array}{l}\text { Leidens- } \\
\text { druck }\end{array}$ & $\begin{array}{l}\text { Behandlungs- } \\
\text { motivation }\end{array}$ & $\begin{array}{l}\text { Behandlungs- } \\
\text { bedarf }\end{array}$ \\
\hline $\begin{array}{l}\text { Behandlungs- } \\
\text { motivation }\end{array}$ & 0,704 & & \\
\hline $\begin{array}{l}\text { Behandlungs- } \\
\text { bedarf }\end{array}$ & 0,596 & 0,569 & \\
\hline $\begin{array}{l}\text { Indikations- } \\
\text { stellung }\end{array}$ & 0,585 & 0,611 & 0,687 \\
\hline
\end{tabular}

Die berechneten Werte sind Kontingenzkoeffizienten

Tab. 4 zeigt die korrelativen Zusammenhänge zwischen Leidensdruck, Behandlungsbedarf, Behandlungsmotivation und Indikationsstellung.

\section{Deskriptive Ergebnisse zu psychischer Belastung/ Komorbidität}

Tab. 5 zeigt den Anteil von Patienten mit auffälligen Werten in den beiden Screening-Instrumenten HADS und BSI.

40,5\% der Patienten zeigen eine psychische Auffälligkeit, gemessen mit der BSI, je über die Hälfte der Patienten (53\% resp. 57,8\%) erreichen auffällige Werte in einer der beiden HADS-Skalen „Angst“ oder „Depression“. Fasst man beide Skalen zusammen, so erreichen über $70 \%$ der Patienten auffällige Werte. Für die HADS muss berücksichtigt werden, dass eine grenzwertige Auffälligkeit als Schwellenwert herangezogen wurde. Damit wurde einer Maximierung der Sensitivität Rechnung getragen. 
Tab. 5 Anteil auffälliger Werte in den Screening-Skalen

\begin{tabular}{|l|l|}
\hline Screening-Skala & Auffällig \\
\hline BSI & $40,5 \%$ \\
\hline HADS-D Depressivität & $53,0 \%$ \\
\hline HADS-D Angst & $57,8 \%$ \\
\hline HADS-D insgesamt auffällig & $71,1 \%$ \\
\hline
\end{tabular}

Beide Screeningverfahren beinhalten zum Teil Redundanzen. Daher wurden die beiden Verfahren zum Kennwert „Psychischer Status“ (siehe Tab. 2) zusammengefasst.

Bereits eine der Subskalen „Angst“ oder „Depression“ mit einem auffälligen Wert wurde als ausreichend erachtet, um für den Kennwert „Psychischer Status“ den Wert „tendenziell auffällig“ (1) zu vergeben. Damit wurde ebenfalls einer Maximierung der Sensitivität Rechnung getragen, genaueres ist Tab. 1 und 2 zu entnehmen.

Die Verteilung des Kennwerts „Psychischer Status“ zeigt, dass $51 \%$ der Patienten entweder in HADS-D oder BSI auffällig sind, und $30 \%$ in beiden Fragebögen. Bei den Patienten, die in beiden Screeningverfahren auffällig sind, kann davon ausgegangen werden, dass eine konsiliarische psychologische/psychotherapeutische Sichtung zumindest unter diagnostischen Gesichtspunkten vonnöten ist.

Validierung des klinischen Urteils über den Zusammenhang von testdiagnostisch erhobenem „Psychischem Status" und klinischem Urteil

Die Gruppe der zu psychologischer Beratung/Psychotherapie indizierten Patienten weist auffälligere Werte im „Psychischen Status" auf als die Gruppe der nicht indizierten Patienten: Der Unterschied zwischen dem mittleren Rang von 62,44 für die Gruppe der nicht indizierten Patienten und dem mittleren Rang von 82,72 für die Gruppe der indizierten Patienten ist hoch signifikant (asymptotische Signifikanz $=0,003$ ). Der korrelative Zusammenhang zwischen Indikationsstellung und „Psychischem Status“ beträgt 0,309 (Spearman) bzw. 0,368 (Pearson) $(\mathrm{P}=0,000)$.

Tab. 6 illustriert die deskriptiven Ergebnisse.

Da Behandlungsmotivation und psychischer Leidensdruck als wichtige Prädiktoren des Kennwertes „Psychischer Status“ vermutet werden, wird weiterhin der Zusammenhang zwischen der ärztlichen Einschätzung (über alle Patienten) für diese Variablen und dem Kennwert „Psychischer Status“ geprüft. Die Spearman-Rang-Korrelation zwischen Leidensdruck und „Psychischem Status“ fällt bei einem Wert von 0,242 signifikant aus (Signifikanz $=0,017$ ). Für Behandlungsmotivation zeigt sich bei einem Wert von 0,127 kein signifikanter Zusammenhang (Signifikanz $=0,213$ ).

Die Indikationsstellung hängt sehr deutlich mit dem klinisch eingeschätzten Leidensdruck und der Behandlungsmotivation zusammen (siehe Tab.5). Damit stellen Leidensdruck und Behandlungsmotivation die Variablen dar, die zwischen dem testdiagnostischen „Psychischen Status“ und der erfolgten Indikationsstellung vermitteln. Falls dies so ist, müsste sich zeigen lassen, dass diejenigen Patienten, die trotz auffälligem psychischen Status nicht zu psychologischen Hilfen indiziert wurden, geringeren Leidensdruck und/oder eine fehlende Behandlungsmotivation aufweisen. Dabei handelt es sich um eine Gruppe von 23 Patienten.
Tab. 6 Kreuztabelle Kennwert „Psychischer Status“ und Indikationsstellung

\begin{tabular}{|llcc|}
\hline & \multicolumn{3}{l}{ Indikationsstellung durch Arzt } \\
\hline Psychischer Status & Nein & Ja & Gesamt \\
\hline 0 & 21 & 4 & 25 \\
\hline 1 & 54 & 14 & 68 \\
\hline 2 & 23 & 19 & 42 \\
\hline gesamt & 98 & 37 & 135 \\
\hline
\end{tabular}

Tab. 7 Behandlungsmotivation der nicht indizierten Patienten mit auffälligem psychischem Status

\begin{tabular}{lccc}
$\begin{array}{l}\text { Behandlungs- } \\
\text { motivation }\end{array}$ & Häufigkeit & Prozent & $\begin{array}{l}\text { Gültige } \\
\text { Prozente }\end{array}$ \\
0 (wenig) & 9 & 39,1 & 60,0 \\
\hline 1 & 4 & 17,4 & 26,7 \\
3 & 1 & 4,3 & 6,7 \\
\hline (sehr hoch) & 1 & 4,3 & 6,7 \\
\hline gültige Werte & 15 & 65,2 & 100 \\
\hline fehlend & 8 & 34,8 & \\
\hline gesamt & 23 & & \\
\hline
\end{tabular}

Kommentar: Der Wert „2“ trat nicht auf.

Tab. 7 zeigt, wie sich die ärztliche Einschätzung der Behandlungsmotivation bei diesen Patienten verteilt: $87 \%$ dieser Patienten mit einem hohen testdiagnostisch ermittelten Kennwert „Psychischer Status“ zeigen eine nach ärztlicher Einschätzung (sehr) geringe Behandlungsmotivation. Dies stützt die Annahme der Behandlungsmotivation als vermittelnder Variable zwischen psychischer Belastung und Indikationsstellung.

\section{Diskussion}

Die pneumologischen Patienten der untersuchten Stichprobe zeigen psychische Belastungen - operationalisiert durch den Kennwert „Psychischer Status“ - in einer Häufigkeit zwischen $30 \%$ und $81 \%$. Legt man eine strenge Interpretation des Kennwerts „Psychischer Status“ an, dann kann von einem Anteil von 30\% pneumologischer Patienten ausgegangen werden, bei denen eine Indikation zur psychologischen/psychotherapeutischen Mitbehandlung gegeben ist. $31 \%$ der Patienten werden von den Ärzten als psychologisch behandlungsbedürftig eingeschätzt und bei $29 \%$ der Patienten erfolgt eine Indikationsstellung zu psychologischer Beratung/Psychotherapie.

Damit bestätigt die vorliegende Studie auch für pneumologische Patienten das von der Freiburger Arbeitsgruppe $[6,13]$ vorgefundene Ausmaß psychischer Beeinträchtigungen bei Patienten in medizinischer Rehabilitation. Sie belegt gleichzeitig die Relevanz der Betrachtung der psychischen Situation in der stationären pneumologischen Rehabilitation. Die eingesetzten Screening-Instrumente können keine diagnostische Differenzierung leisten zwischen psychischen Störungen nach ICD mit eigenständigem Krankheits- und Behandlungswert (z. B. Angsterkrankungen, Störungen aus dem depressiven Formenkreis) und psychischen Beeinträchtigungen, die als abhängig von der pneumologischen Erkrankung zu fassen sind (z.B. Angst vor Atemnot und nachfolgend phobische Vermeidung spezifischer Situationen oder depressive Anpassungsreaktion bei eingeschränkter sozialer Teilhabe). Bei einer solchen Differenzierung dürfte sich 
der Anteil der Patienten mit während des Aufenthalts vorliegender behandlungsbedürftiger psychischer Störung mit eigenständigem Krankheitswert geringer darstellen. Dies zeigen auch Ergebnisse von Härter u. Mitarb. [13].

Gleichwohl ist eine Einbeziehung krankheitsabhängiger psychischer Beeinträchtigungen im Rahmen eines biopsychosozialen Rehabilitationsverständnisses und unter der Annahme, dass psychische Komorbiditäten den Verlauf der pneumologischen Erkrankung ungünstig beeinflussen können [1], geboten. Ihre Erhebung und fachgerechte Behandlung ist Teil einer leitliniengemäßen Rehabilitation [14,15].

Unter diesem Gesichtspunkt weist der Vergleich zwischen der Bedarfsschätzung aufgrund des Screenings mit der Indikation zu psychologischen Hilfen in der betrachteten pneumologischen Reha-Klinik auf eine solide Validität dieser Indikationspraxis hin: Die Gruppe, bei der ärztlicherseits eine psychologische/psychotherapeutische Mitbehandlung indiziert wurde, unterscheidet sich signifikant im Kennwert „Psychischer Status“ von denjenigen Patienten, bei denen diese Indikationsstellung nicht erfolgte. Die ärztliche Indikationsstellung erklärt sich weiter gut aus den Variablen psychischer Leidensdruck und Behandlungsmotivation.

Die Indikationsstellung integriert als pragmatische Entscheidung des behandelnden Arztes die Einschätzung der Behandlungsmotivation des Patienten, woraus sich Diskrepanzen zwischen Test- und klinischem Urteil erklären lassen.

Die Ergebnisse zeigen die Notwendigkeit der regelhaften diagnostischen Erhebung der psychischen Situation bei pneumologischen Rehabilitationspatienten und die Bedeutung der Vorhaltung von psychologischen und psychotherapeutischen Ressourcen, um psychische Belastungen und psychische Komorbidität angemessen behandeln zu können.

\section{Literatur}

1 Bengel J, Jäckel WH: Zu Prävalenz und Behandlungsbedarf psychischer Belastungen in der Rehabilitation. Die Rehabilitation 2002; 41: 355 356

2 BTS statement: Pulmonary rehabilitation. Thorax 2001; 56 (11): 827 834

3 Bundesversicherungsanstalt für Angestellte: Leitlinien zur Rehabilitationsbedürftigkeit bei Krankheiten der Atmungsorgane - für den beratungsärztlichen Dienst der BfA. Berlin 2004, Internet: http://www.bfa.de

4 Donner CF, Muir JF: Rehabilitation and Chronic Care Scientific Group of the European Respiratory Society. Selection criteria and programmes for pulmonary rehabilitation in COPD patients. Eur Respir J 1997; 10: 744-57

5 Härter M: Ätiologie psychischer Störungen bei chronischen körperlichen Erkrankungen. Die Rehabilitation 2002; 41: 357-366

6 Härter M, Baumeister H, Hahn D, Bengel J: Psychische Störungen bei Rehabilitanden mit chronischen körperlichen Erkrankungen. In: Verband Deutscher Rentenversicherungsträger (Hrsg). Selbstkompetenz. Weg und Ziel der Rehabilitation. 13. Rehabilitationswissenschaftliches Kolloquium vom 8. - 10. März 2004 in Düsseldorf (S. 306 - 307). DRV Schriften, Bd. 52

7 Mayou R, Sharpe MC: Psychiatric illnesses associated with physical disease. In: Bramley I (ed). Baillière's Clinical Psychiatry (Vol 1/No 2). Cambridge: University Printing House 1995: 201 - 223

8 Yohannes AM, Baldwin RC, Connolly MJ: Depression and anxiety in elderly outpatients with chronic obstructive pulmonary disease: prevalence, and validation of the BASDEC screening questionnaire (abstract). 2000 [online]. Verfügbar unter: http://www.ncbi.nlm.nih.gov/

9 Schwiersch M: Psychologischer Support in der pneumologischen Rehabilitation. Was ist gesichert? Atemwegs- und Lungenerkrankungen 2004; 30/9: 450-455

10 Härter M: Diagnostik in epidemiologischen Untersuchungen. In: Stieglitz RD, Baumann U, Freyberger HJ (Hrsg). Psychodiagnostik in klinischer Psychologie (2., überarbeitete und erweiterte Auflage). Stuttgart: Thieme 2001: 339-350

11 Franke GH: Brief Symptom Inventory (BSI) von L.R. Derogatis. Deutsche Version. Testmappe. Göttingen: Beltz 2001

12 Hermann Ch, Buss U: HADS-D; deutsche Version; ein Fragebogen zur Erfassung von Angst und Depressivität in der somatischen Medizin; Testdokumentation und Handanweisung. Bern: Huber 1995

13 Härter M, Baumeister H: Psychische Komorbidität bei somatischen Erkrankungen: Präavalenz, Ätiologie und Diagnostik. In: Faller H (Hrsg). Psychotherapie bei somatischen Erkrankungen. Stuttgart: Thieme 2005: 7-17

14 Nationale Versorgungsleitlinie Asthma: http://www.awmf-online.de bzw. www.versorgungsleitlinien.de/themen/asthma. 2006

15 Nationale Versorgungsleitlinie COPD: http://www.awmf-online.de bzw. www.versorgungsleitlinien.de/themen/copd. 2006 